\title{
Gingipain-responsive thermosensitive hydrogel loaded with SDF-1 facilitates in situ periodontal tissue regeneration
}

Shiyue Liu ${ }^{a}+$, Ya-nan Wang ${ }^{b,+}$, Baojin Ma ${ }^{a}$, Jinlong Shao ${ }^{a}$, Hongrui Liu ${ }^{a}$, Shaohua Ge ${ }^{a^{*}}$

a Department of Periodontology, School and Hospital of Stomatology, Cheeloo College of Medicine, Shandong University \& Shandong Key Laboratory of Oral Tissue Regeneration \& Shandong Engineering Laboratory for Dental Materials and Oral Tissue Regeneration, Jinan, Shandong, 250012, China

b Department of Implantology, School and Hospital of Stomatology, Cheeloo College of Medicine, Shandong University \& Shandong Key Laboratory of Oral Tissue Regeneration \& Shandong Engineering Laboratory for Dental Materials and Oral Tissue Regeneration, Jinan, Shandong, 250012, China

†These authors contributed equally.

${ }^{*}$ Corresponding author:

Shaohua Ge, No.44-1 Wenhua Road West, 250012, Jinan, Shandong, China, Tel.: +86-531-88382123, Fax: +86 53188382923, E-mail: shaohuage@sdu.edu.cn 


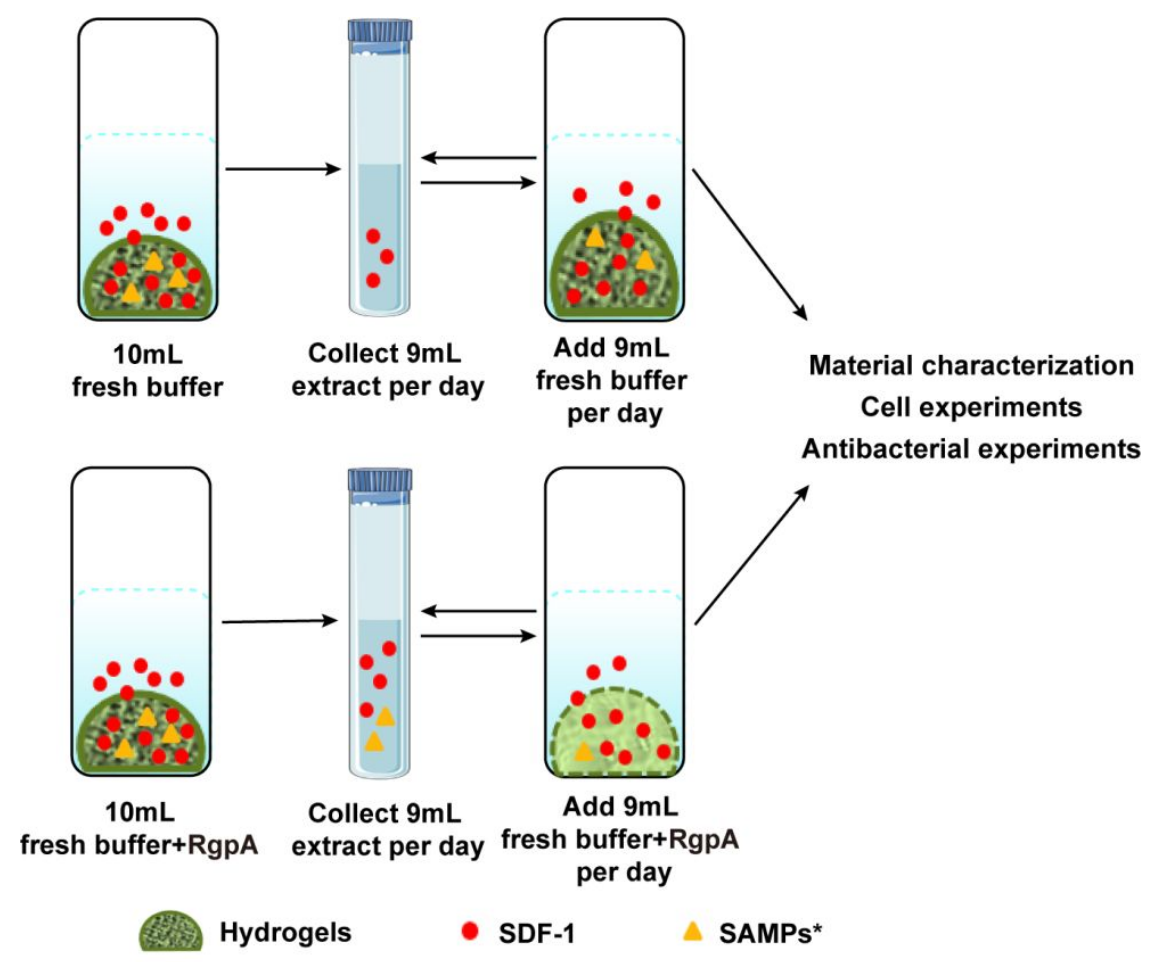

Figure S1. Schematic illustration of hydrogel extracts acquisition system. Application scenario includes the characterization of hydrogel materials, cell migration and osteogenic differentiation detection, and antibacterial experiments. Fresh buffer contains PBS, DMEM, and BHI. SDF-1 represents stromal cell-derived factor-1. SAMP* is composed of KSL (KKVVFKVKFK-NH2, SAMP used in this article) and 5 amino acid residues on each side.
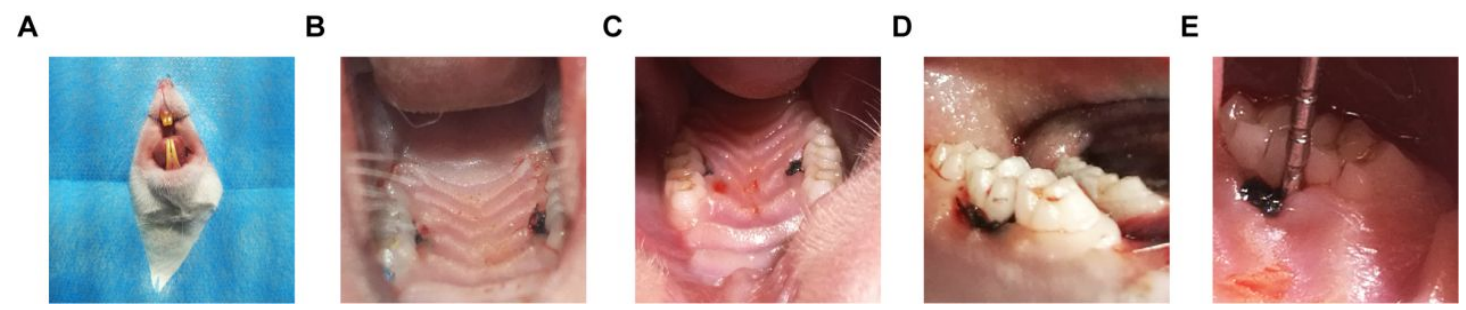

Figure S2. Schematic illustration of establishing the rat periodontitis model. (A) A Wistar rat was in supine position after anesthesia. (B) The 4-0 line was tied around the maxillary second molar, $\mathrm{T}=0$ day. (C-E) Periodontal status of the maxillary second molar 14 days after modeling, redness gum bleeding on probing. 


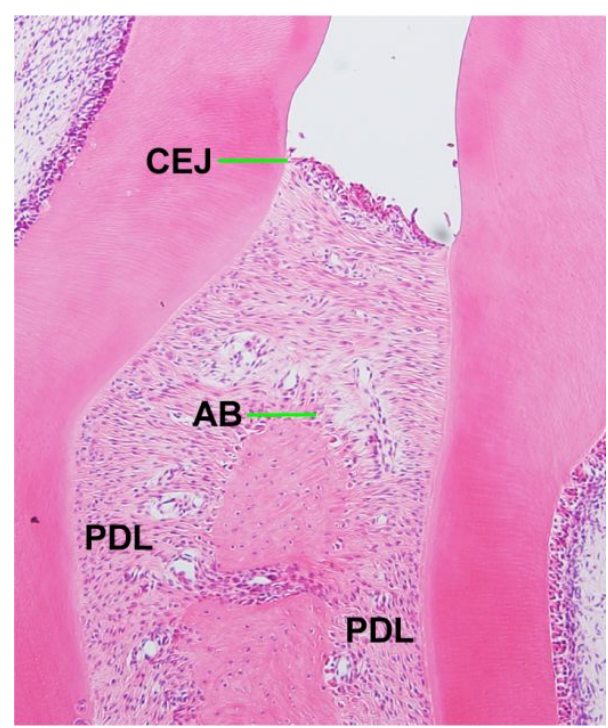

Figure S3. Schematic illustration of the main periodontal structures between the maxillary first molar and the second molar stained by H\&E. CEJ: cemento-enamel junction. AB: alveolar bone crest. PDL: Periodontal ligament. 
A

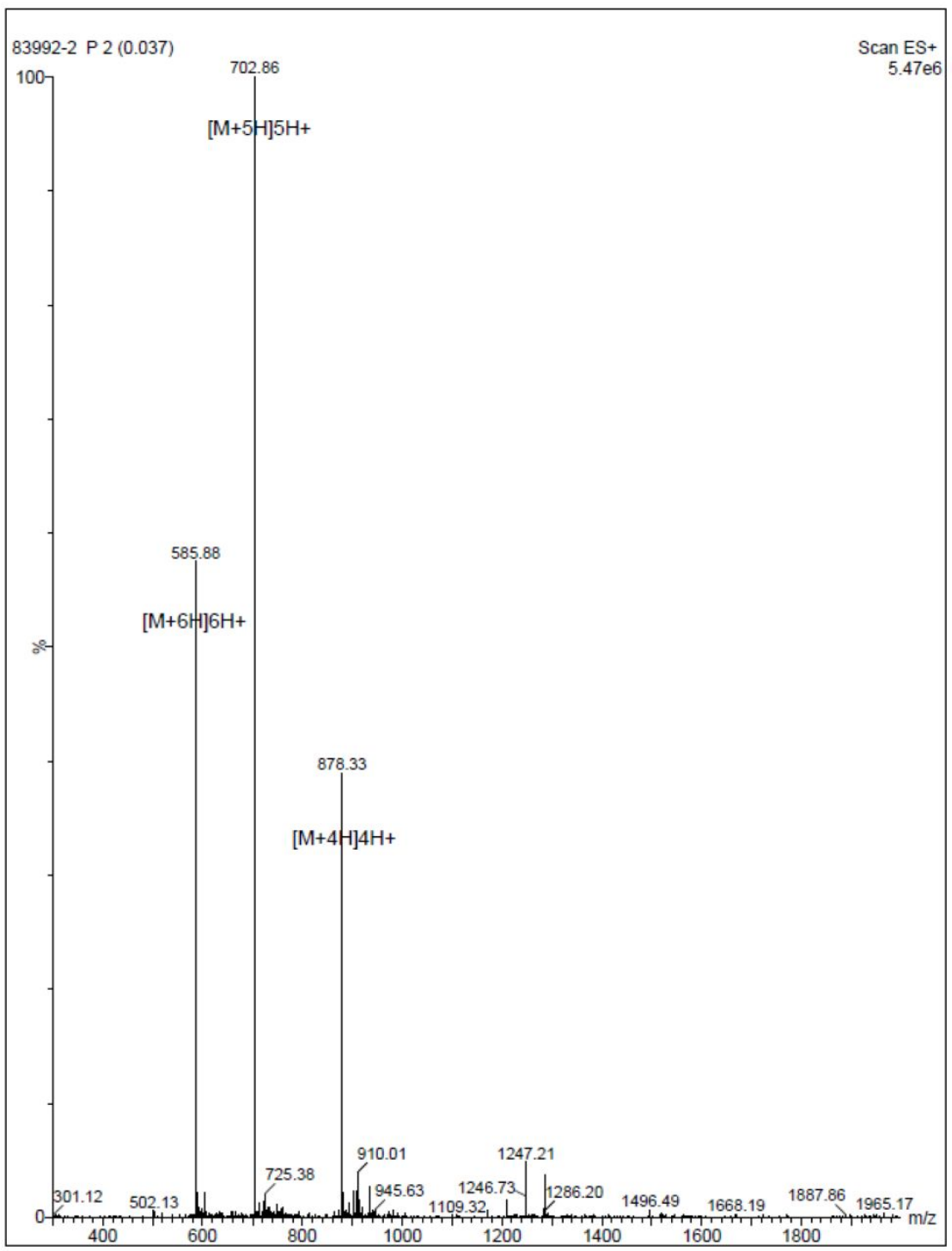

B

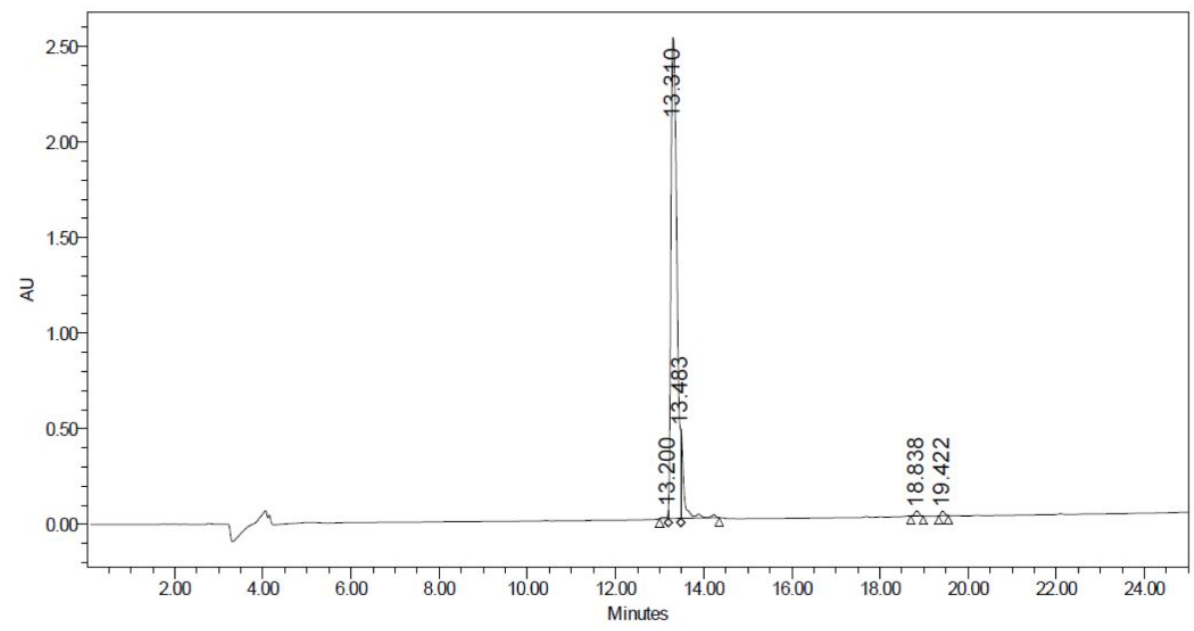

Figure S4. Characterization of molecular weight (A) and purity (B) of synthetic functional peptide module (FPM). 


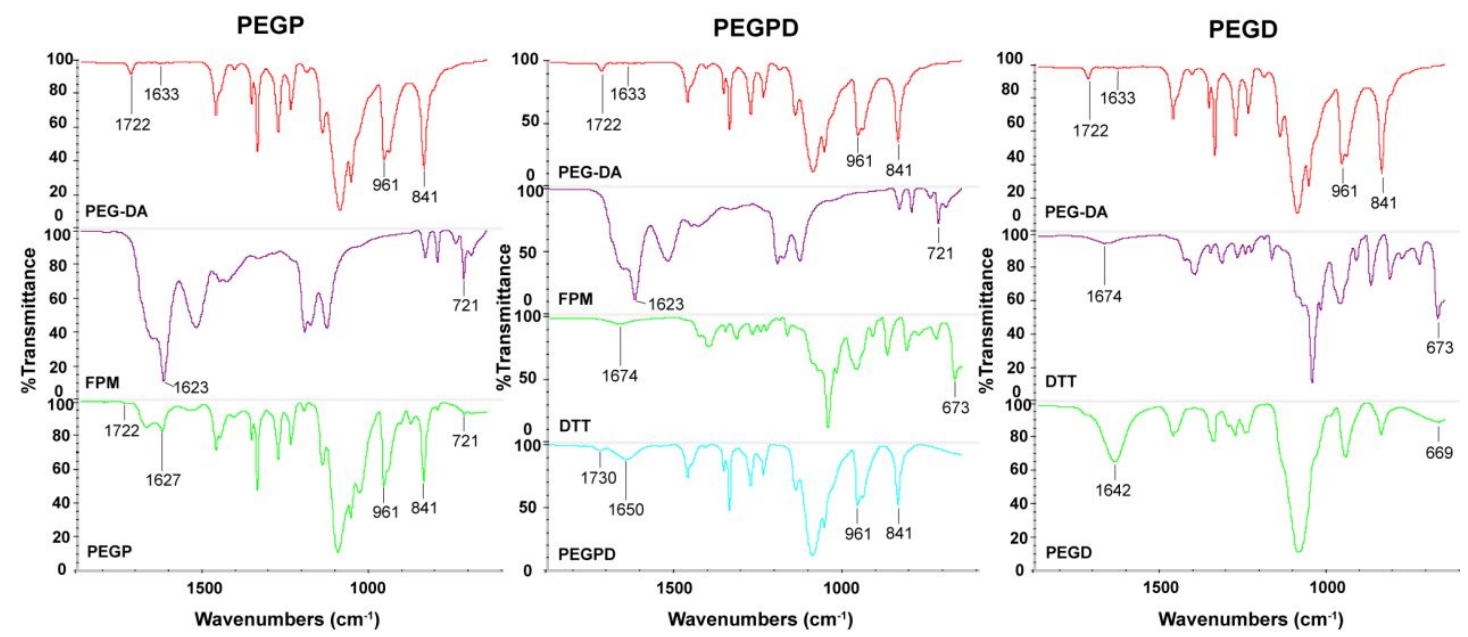

Figure S5. Infrared spectra of PEG-DA, FPM, DTT, and three kinds of hydrogels. 

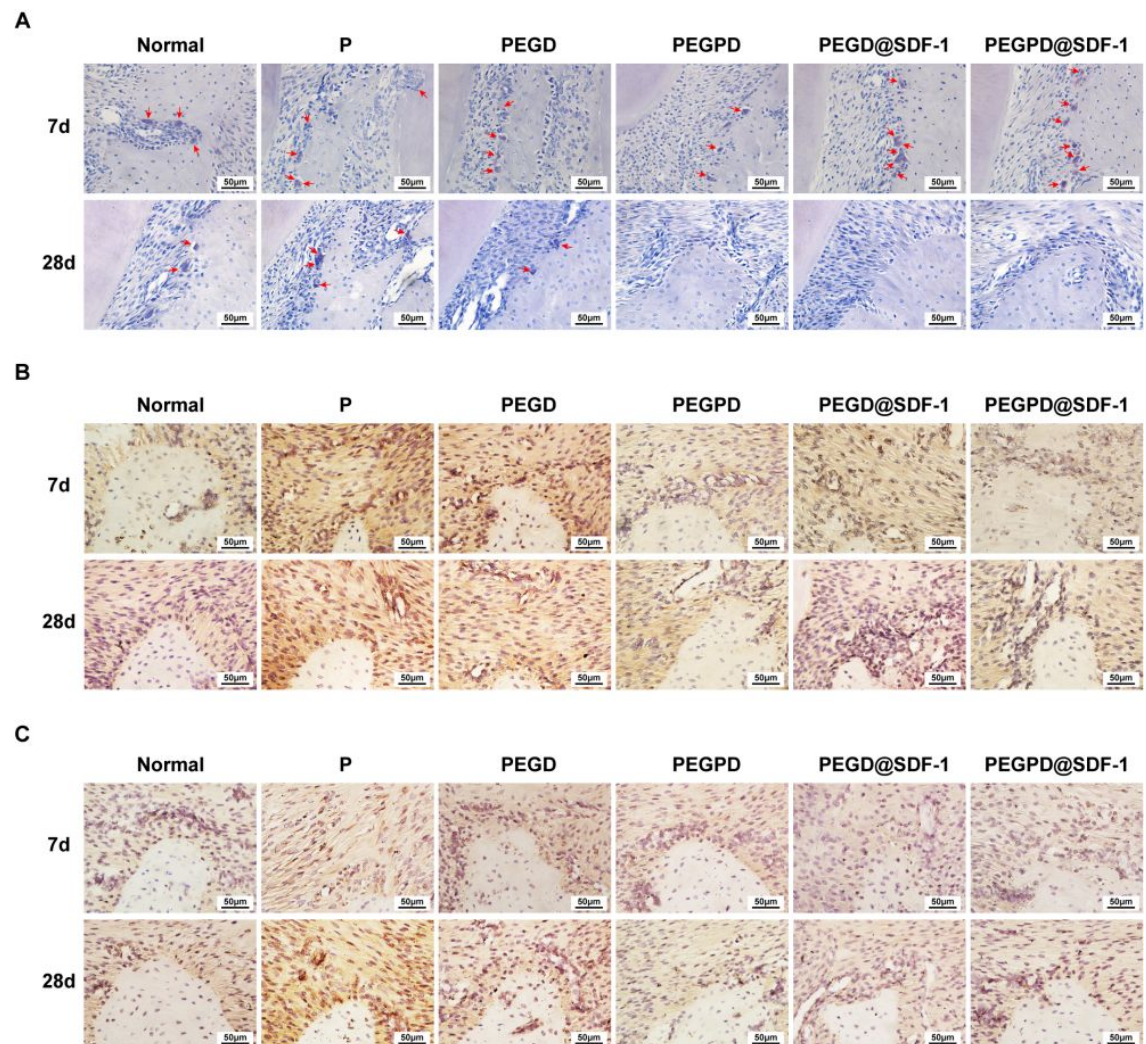

D

$\mathrm{E}$

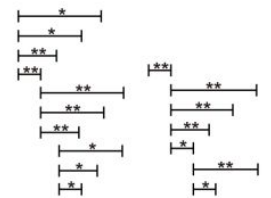

$\mathbf{F}$
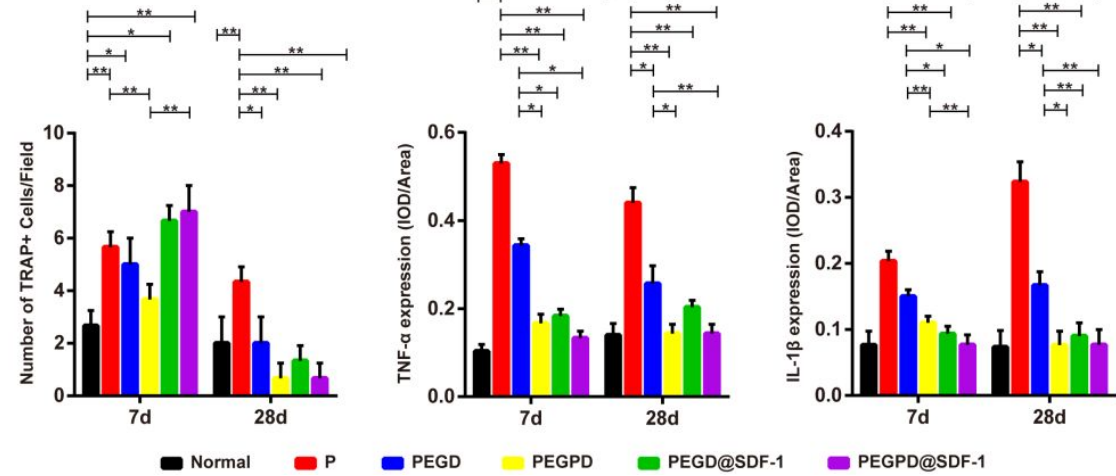

Figure S6. Effect of PEGPD@SDF-1 hydrogel on inflammation control in vivo. (A) TRAP staining representing osteoclastogenesis 7 and 28 days after hydrogel injection. (B) Immunohistochemical staining of TNF- $\alpha$ expression 7 and 28 days after hydrogel injection. (C) Immunohistochemical staining of IL-1 $\beta$ expression 7 and 28 days after hydrogel injection. (D) Counting for the number of TRAP+ cells. (E) Quantitative analysis of mean integral optical density (IOD) for TNF- $\alpha$. (F) Quantitative analysis of mean integral optical density (IOD) for IL-1 $\beta$. Data were presented as mean $\pm \mathrm{SD}, \mathrm{N}=6 .{ }^{*} p<0.05,{ }^{* *} p<0.01$. 


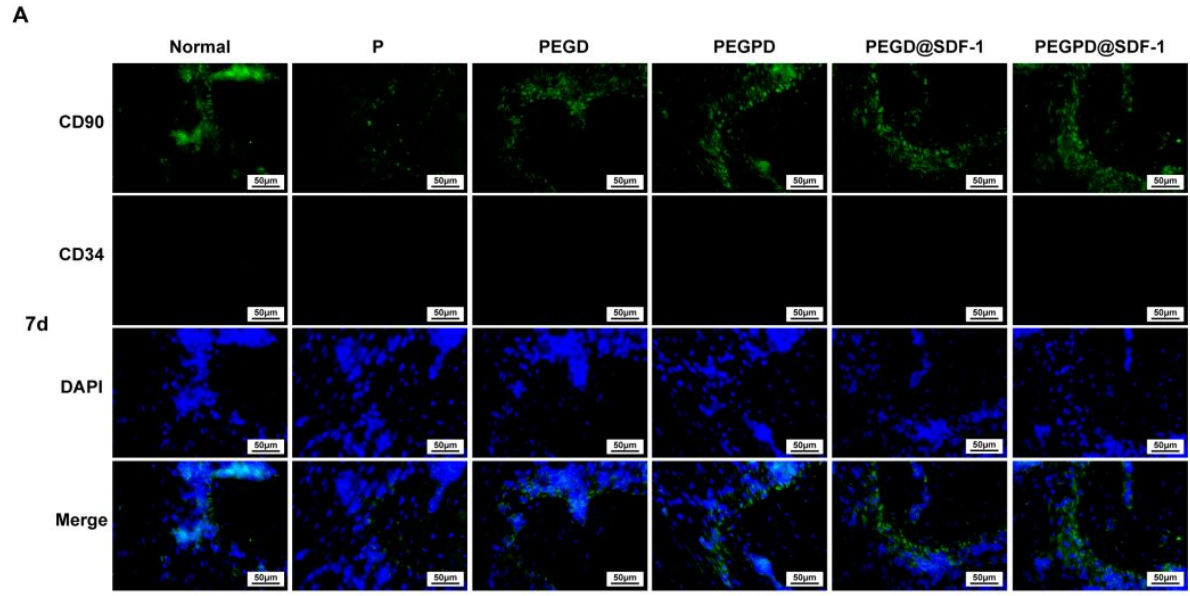

B

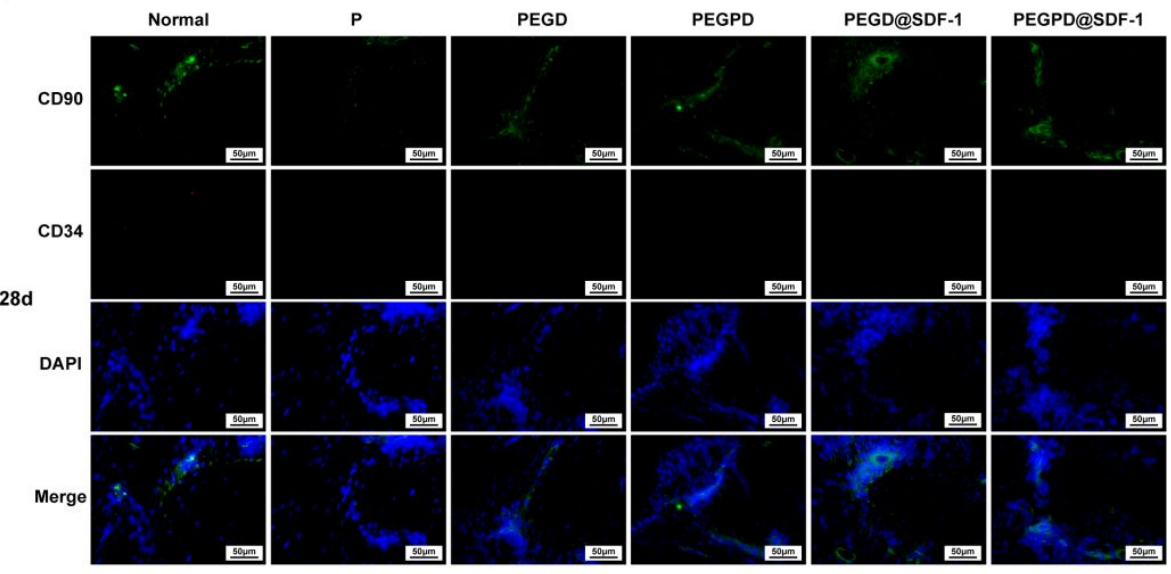

C

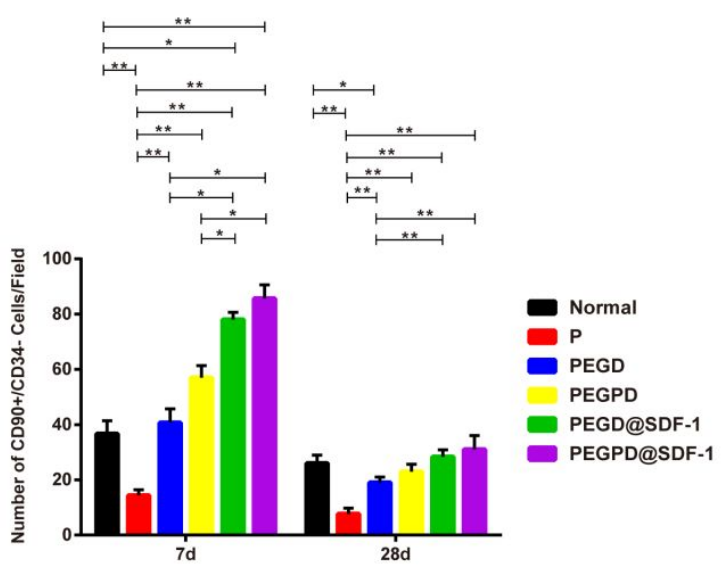

Figure S7. Effect of PEGPD@SDF-1 hydrogel on the recruitment of stem cells in vivo. (A-B) Immunofluorescent staining for CD90+/CD34- stromal cells in the periodontal tissue between the maxillary first molar and second molar 7 and 28 days after hydrogel injection. (C) Counting for the number of CD90+/CD34- stromal cells (CD90+ cells: Green, CD34+ cells: Red). Data were presented as mean $\pm \mathrm{SD}, \mathrm{N}=6 .{ }^{*} p<0.05,{ }^{* *} p<0.01$. 